\title{
Neonatal sepsis in South Asia: huge burden and spiralling antimicrobial resistance
}

\author{
M Jeeva Sankar and colleagues call for urgent action to improve quality of care at birth and \\ implement antimicrobial stewardship in health facilities in South Asia to reduce neonatal deaths \\ from sepsis
}

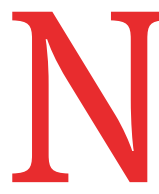

eonatal sepsis, a systemic infection in the first 28 days of life, encompasses bloodstream infections, meningitis, and pneumonia. It is the third most common cause of deaths among neonates, accounting for 225000 deaths globally every year. ${ }^{1}$

South Asia and sub-Saharan Africa have the highest burden of neonatal sepsis in the world. Of the total sepsis related neonatal deaths in 2013, 38.9\% occurred in South Asia. ${ }^{12}$ Poverty, low coverage of effective interventions, including facility births, and gross inequities in delivery of healthcare ${ }^{3}$ contribute to this situation. We review the available literature (box 1) to draw attention to the burden of neonatal sepsis, the pathogen profile, and the extent of antimicrobial resistance in South Asia, and propose priority actions for policymakers and health professionals in the region.

\section{Paucity of high quality data}

We found no data on neonatal sepsis from Afghanistan, Bhutan, Maldives, and little

\section{KEY MESSAGES}

- The incidence of neonatal sepsis in South Asia is 4 to 10 times higher than that in developed countries

- Unlike high income countries with a predominance of group B streptococci, Gram negative organisms predominate, possibly indicating horizontal transmission of infections from the environment and healthcare providers

- About $50-88 \%$ of common isolates from the health facilities are resistant to first line antibiotics-ampicillin and gentamicin

- Simple, evidence based interventions can help, such as better asepsis, hand hygiene, and exclusive breastfeeding and establishing antimicrobial stewardship programmes from Sri Lanka. While the neonatal health indicators of three of these four countries are reasonably good, ${ }^{3}$ the paucity of data from war torn Afghanistan is a cause for concern.

More importantly, the data from the four countries with the highest burden of neonatal sepsis (India, Pakistan, Nepal, and Bangladesh) are of poor quality. With the exception of studies such as the Young Infant Study ${ }^{4}$ ANISA, ${ }^{5}$ and the multisite study from the Delhi Neonatal Infections Study ${ }^{7}$ collaboration, most studies are retrospective, lack rigorous methods and standard definitions, and have used passive surveillance to identify cases. There is therefore an inherent risk of underestimating the burden of sepsis in the region.

\section{High incidence of sepsis}

The pooled incidence of culture positive sepsis in hospital based reports from South Asia is 15.8 per 1000 live births (95\% CI 12.7 to $18.8, \mathrm{n}=15$ reports). This is about twofold to fourfold higher than that reported in high income countries such as England and the United States. ${ }^{89}$ The incidence does not seem to have declined in the last decade. ${ }^{10}$ About a third of neonates with culture positive sepsis died as a result (median case fatality rate $34.4 \%$ ). Table 1 presents incidence and case fatality rates by country.

Sepsis is categorised into early onset sepsis (onset within 72 hours of birth) and late onset sepsis (beyond 72 hours). Early onset sepsis is thought to be caused by pathogens vertically transmitted from mothers while late onset sepsis is attributed to pathogens acquired horizontally from the environment or care givers, or both. About $62 \%$ of the infections in South Asia occur in the first 72 hours of life, roughly translating into an incidence of 9.8 per 1000 live births. This is 10-fold higher than the incidence of early onset sepsis reported in a large nationwide study in the United States. ${ }^{9}$

Neonatal sepsis is classified as culture positive or culture negative, depending on

\section{Box 1 Sources and methods}

We searched PubMed and Web of Science for literature published between January 2000 and August 2018 using the search terms: (newborn OR neonate) AND (sepsis OR infection OR antibiotic $O R$ antimicrobial). The results were filtered for South Asian countries. Bibliographies of full text articles and published systematic reviews were also searched to identify additional articles.

We identified 2699 and 220 articles from PubMed and Web of Science, respectively, and 19 additional articles from reference lists of identified articles. After removing duplicates, we screened 2768 articles and reviewed 223 full text articles. Finally, 109 studies were included: 69 from India, 16 from Pakistan, 7 from Bangladesh, 14 from Nepal, 1 from Sri Lanka, and 2 multi-country studies (Young Infant Study ${ }^{4}$ and Aetiology of Neonatal Infections in South Asia $(\text { ANISA })^{5}$ ) covering Bangladesh, India, and Pakistan). Because the datasets belonging to different time periods and/or different patient populations in a given study were considered unique, we had a total of 123 datasets from 109 studies.

Two authors (SS and SC) extracted information from the relevant studies. Most studies were hospital based, single centre studies that reported data on neonates with suspected sepsis or laboratory based studies reporting bacteriological profile and antimicrobial resistance of cultures received from neonatal intensive care units. We identified only eight relevant community based studies. Random effects meta-analysis was done to pool the results, if applicable, using the "metan" command in Stata 15.1 (StataCorp, College Station, TX). Pooling of rates was done separately for the hospital based and community based studies. See appendix on bmj.com for details of the methods and studies included. 


\begin{tabular}{|c|c|c|c|c|c|c|}
\hline & India & Pakistan & Bangladesh & Nepal & Sri Lanka & Total \\
\hline No of studies (No of isolates) & $64(18761)$ & $16(3557)$ & $6(584)$ & $14(1325)$ & $1(9)$ & $101^{*}(24244)$ \\
\hline $\begin{array}{l}\text { Incidence }(95 \% \mathrm{Cl}) \text { of culture positive } \\
\text { sepsis, per } 1000 \text { live births; No of } \\
\text { studies }\end{array}$ & $\begin{array}{l}16(12.8 \text { to } 19.2) \\
n=14\end{array}$ & NA & NA & $\begin{array}{l}11.6(18.4 \text { to } 14.7) ; \\
n=1\end{array}$ & $\begin{array}{l}\text { 13.6/1000 patient } \\
\text { days; } n=1\end{array}$ & $\begin{array}{l}15.7 \text { (12.7 to } 18.8) ; \\
n=15\end{array}$ \\
\hline $\begin{array}{l}\text { Case fatality rate }(95 \% \mathrm{Cl}) ; \text { No of } \\
\text { studies }\end{array}$ & $\begin{array}{l}34.4(33 \text { to } 35.7) \\
n=14\end{array}$ & $\begin{array}{l}30.9(25.7 \text { to } \\
36.2) ; n=2\end{array}$ & $\begin{array}{l}19.1 \text { (11.7 to } 26.5) ; \\
n=2\end{array}$ & $\begin{array}{l}64.7(54.3 \text { to } 75) ; \\
n=1\end{array}$ & NA & $\begin{array}{l}34.4(33.1 \text { to } 35.6) ; \\
n=19\end{array}$ \\
\hline \multicolumn{7}{|l|}{$\begin{array}{l}\text { \% Infecting organism }(95 \% \mathrm{Cl}) \text {; No of } \\
\text { studies }\end{array}$} \\
\hline Klebsiella sppt & $\begin{array}{l}53.6(50.7 \text { to } 56.5) ; \\
n=21\end{array}$ & NA & NA & $\begin{array}{l}33(3.0 \text { to } 63.0) \\
n=1\end{array}$ & 60 & $\begin{array}{l}53.3(50.4-56.2) ; \\
n=22\end{array}$ \\
\hline Escherichia colit & $\begin{array}{l}42.2(38.5 \text { to } 46) ; \\
n=16\end{array}$ & NA & NA & $\begin{array}{l}50(1.0 \text { to } 98.0) \\
n=1\end{array}$ & NA & $\begin{array}{l}42.2(38.6 \text { to } 46.2) ; \\
n=17\end{array}$ \\
\hline $\begin{array}{l}\text { Meticillin resistant Staphylococcus } \\
\text { aureus }\end{array}$ & $\begin{array}{l}43.2(39.9 \text { to } 46.5) ; \\
n=17\end{array}$ & $\begin{array}{l}61(49.1-72.8) \\
n=1\end{array}$ & NA & $\begin{array}{l}26(12.8 \text { to } 39.3) \\
n=3\end{array}$ & NA & $\begin{array}{l}46.5(41.9 \text { to } 51.1) \\
n=21\end{array}$ \\
\hline
\end{tabular}

Data represent pooled proportions $(95 \% \mathrm{Cl})$, unless stated otherwise. $\mathrm{NA}=$ not available

*Community based studies $(n=8)$ are not included in table.

tExtended spectrum $\beta$ lactamase.

isolation of pathogen(s) from blood or other sterile fluids. Based on the available data, this review focuses on the burden of culture positive sepsis, which forms only part of the total number of sepsis cases. Because of the difficulty in obtaining an adequate volume of blood in preterm neonates and the low levels of bacteraemia, blood cultures tend to be sterile in many neonates. The culture positive versus culture negative sepsis ratio ranges from 1:6 to 1:16 in high income countries. ${ }^{11}$ The ratio is likely to be more skewed towards culture negative sepsis in South Asia, given the poor microbiological laboratory support in most units. The burden of total neonatal sepsis is therefore likely to be much higher.

The proportion of neonates with culture positive sepsis in community based studies was lower-12.3 per 1000 live births (95\% CI 8.4 to 16.2 ). However, this was influenced by the ANISA study ${ }^{5}$ which used a statistical model incorporating the results of molecular assays to estimate the incidence of bacterial sepsis. If only conventional blood culture positive results are included, the pooled risk of sepsis was much lower-5.5 per 1000 live births (95\% CI 2.4 to 8.6).

\section{Unique pathogen profile}

The pathogen profile in South Asia is different from that found in high income countries. There is a predominance of Gram negative pathogens ( $>60 \%)$ and a low prevalence of group B streptococci in South Asia, compared with a high incidence of group B streptococci in high income countries. $^{710}$

Among isolates from hospital settings $(n=24273)$, Gram negative organisms (63\%) were the most common, with Klebsiella spp (23\%), Escherichia coli (14\%), and Acinetobacter spp (8\%) being the top three. The most common Gram positive organisms were Staphylococcus aureus (20\%) and Coagulase negative Staphylococci (9\%). Gram negative organisms were associated with higher case fatality (pooled proportion 26.7\%; 95\% CI 0 to $41 \%$ ) than Gram positive organisms (11.9\%; 95\% CI 10.5 to $13.3 \%$ ). Among the 703 isolates from community settings,
Klebsiella spp (25\%), E coli (15\%), and $S$ aureus (12\%) were the most common.

The predominance of Gram negative pathogens in South Asia suggests that the transition from Gram negative pathogens to Gram positive organisms such as group B streptococci that happened five to six decades ago in developed countries ${ }^{10}$ largely because of improved aseptic routines, including hand hygiene in neonatal intensive care units-is yet to take place in South Asia.

There was a striking similarity between the pathogen profile of early onset and late onset sepsis (see supplementary fig 2 on bmj.com). This challenges the assumption of attributing early onset sepsis to vertical transmission from the mothers. Although it is possible that the pregnant women in South Asia are colonised with pathogens normally found in the hospitals (such as Klebsiella), it is more probable that the source of infection in early onset sepsis is the unhygienic practices in the labour rooms and neonatal intensive care units. Identifying the source and transmission pathways of common pathogens of early

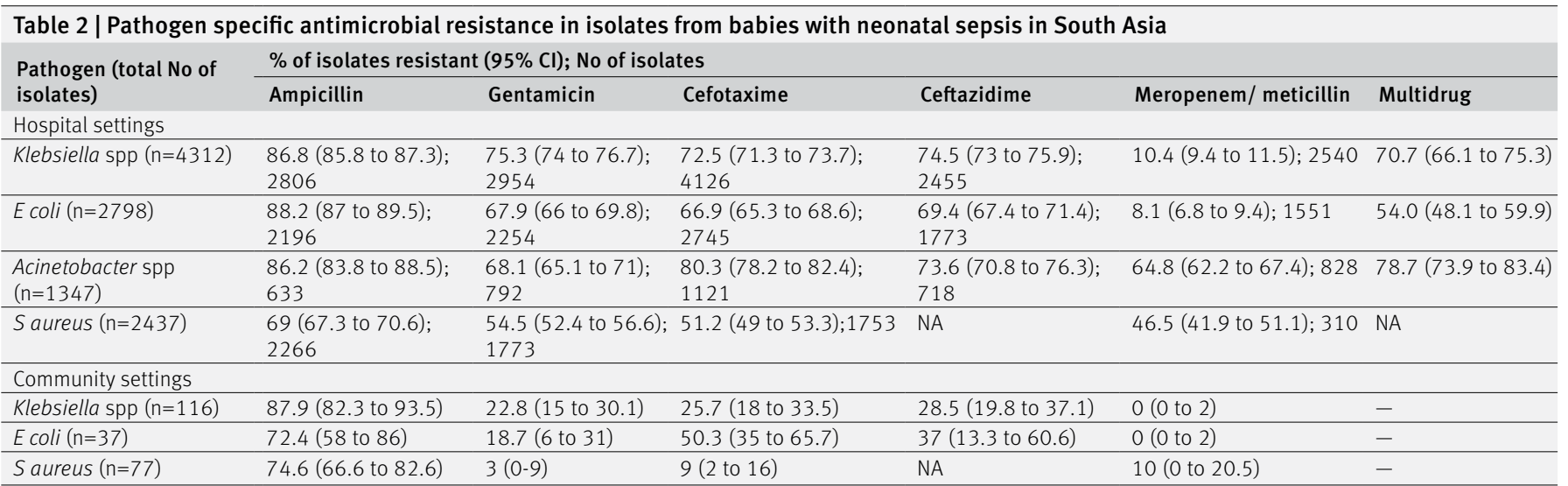

Data represent pooled proportion $(95 \% \mathrm{Cl}) ; n=$ number of isolates tested, unless stated otherwise. $\mathrm{NA}=$ not applicable. 


\section{Box 2 Research priorities for neonatal sepsis in South Asia}

- Establish a subnational/national surveillance database to evaluate and monitor the burden of neonatal sepsis, sepsis related mortality, and antimicrobial resistance

- Implementation research-quality improvement initiatives-to scale up the coverage of known interventions

- Identify the source of infection and transmission pathways of common pathogens

- Evaluate the impact of introducing antimicrobial stewardship programmes at different levels of health facilities

- Develop and validate point-of-care diagnostic method(s) for rapid and accurate diagnosis of sepsis

onset sepsis is essential to determine the appropriate steps to prevent infection that will help to reduce the high burden of mortality associated with early onset sepsis in the region.

\section{Spiralling antimicrobial resistance}

Antimicrobial resistance has worsened in the last decade, rendering most antibiotics obsolete. Resistance to even "reserve" antibiotics has increased-50-70\% of the common Gram negative isolates are now multidrug resistant. Table 2 presents antimicrobial resistance patterns found in common pathogens in hospital and community settings in South Asia.
The common pathogens from hospital based studies uniformly exhibit a high degree of resistance to first line drugs recommended by the World Health Organization-namely, ampicillin, gentamicin, and third generation cephalosporins such as cefotaxime (table 2). ${ }^{6}$ However, most were susceptible to WHO classified "watch group"6 antibiotics, such as meropenem (see suppl table 1 on bmj.com). About half (pooled proportion $46.5 \%$; $95 \%$ CI 41.9 to 51.1 ) of $S$ aureus isolates were meticillin resistant, but most remained susceptible to watch group antibiotics such as vancomycin. The varied pathogens with high resistance

Team
$\bullet$ Neonatologist $\bullet$ Senior nursing officer
$\bullet$ Microbiologist $\bullet$ Statistician/public health expert

Strategies

- Scale up infection control practices

- Improve microbiology lab capacity (automated cultures)

- Standard operating procedures:

Obtain blood culture before first dose of antibiotics

When to screen and when to treat for sepsis

- Unit protocol:

Antibiotic policy based on culture reports of last 6-12 months

Stop or de-escalate to narrow spectrum antibiotics based on culture report

- Prior authorisation from antimicrobial stewardship programme team for "reserve" antibiotics

\section{Audit and feedback}

\section{- Measure: proportion}

In whom cultures obtained before start of antibiotics

Receiving "reserve" antibiotics

Receiving right drug, right dose, and right duration

Not receiving antibiotics in sick newborn care unit

- Measure: days of therapy (DOT) of common antibiotics

- Prospective audit and feedback to staff

Fig 1 | Proposed model of antimicrobial stewardship programme for health facilities in South Asia. Adapted from Ramasethu 2017; Patel 2012; Cantey 2014 patterns preclude the use of intrapartum antibiotic prophylaxis to prevent vertically transmitted infection. In contrast, the antimicrobial resistance profile in the community based studies was not so high: although resistance to ampicillin was high, resistance to gentamicin and third generation cephalosporins was low.

If the apparent dichotomy in antimicrobial resistance between the hospital based and community based studies is real, health facilities in the region should review their antibiotic policy to prevent misuse of antibiotics. The high antimicrobial resistance brings into focus the overuse of antibiotics in neonates with culture negative sepsis. The ANISA study showed that many neonates with negative blood cultures had viral infections. ${ }^{5}$ The low case fatality in neonates with culture negative sepsis (only one fifth of that of culture positive sepsis) in the multisite study from Delhi ${ }^{7}$ also suggests that many of these neonates either had viral infections or did not have sepsis at all. More reliable and accurate point-of-care diagnostic method(s) are needed to rule out sepsis, thereby preventing indiscriminate use of antibiotics in neonatal intensive care units.

The low antimicrobial resistance in the community allows healthcare providers in primary and even secondary level facilities to use first line antibiotics in neonates with sepsis. Steps to prevent misuse of antibiotics in other sectors such as agriculture and restriction of over-thecounter antibiotics ${ }^{1516}$ would ensure that the antimicrobial resistance rates remain low in community settings.

\section{Next steps}

\section{Surveillance and research}

We need reliable data to track progress on neonatal sepsis in the region to enable benchmarking and cross-learning and inform policy making. A regional surveillance database-possibly under the umbrella of the South Asian Association for Regional Cooperation-could provide such a mechanism, alongside surveillance databases within each country. This could begin with passive surveillance using uniform definitions and be followed up by active surveillance. Box 2 lists the major research priorities in the region.

\section{Quality of care and antimicrobial stewardship in hospitals}

Most health facilities in the region continue to be the "hot beds" of infection transmission in sick and vulnerable neonates. ${ }^{10}$ Lack of essential equipment and supplies including soap, sinks, running water, and 


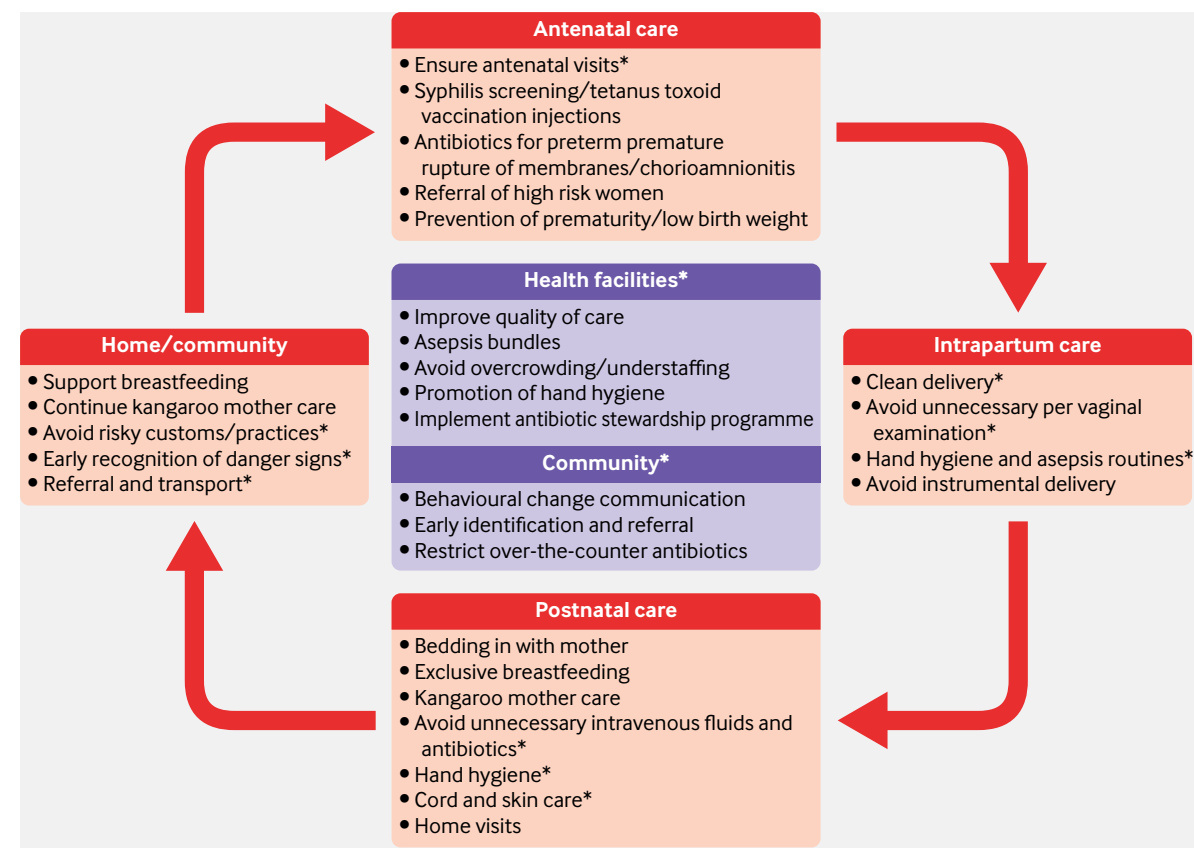

Fig 2 | Key interventions to reduce the burden of sepsis and antimicrobial resistance in South Asia. *Indicates interventions that are more relevant to the region

disposables; overcrowding and understaffing; and suboptimal disinfection practices increase the risk of horizontal transmission of infections from the labour rooms or neonatal intensive care unit. ${ }^{10}$ The twofold greater risk of sepsis in hospital based studies compared with community based studies suggests that horizontal transmission-that is, healthcare associated infections-plays a major part in the high incidence of neonatal sepsis in the region. Selective referral of high risk neonates to hospitals may also account for this. With increasing rates of facility births, the burden of neonatal sepsis is bound to increase unless radical measures to improve the quality of care are implemented.

Antimicrobial stewardship programmes must be implemented to rationalise antibiotic use. Policy makers and other stakeholders should develop guidelines and toolkits to facilitate implementation of these programmes across health facilities at different levels of the health system. Steps should be simplified and continuous monitoring and auditing done to inform healthcare providers of the progress made. Figure 1 depicts a simplified model of antimicrobial stewardship programmes for use in health facilities in the region. ${ }^{12-14}$

\section{Scale up effective interventions}

The Every Newborn series on quality of care at birth identified that scaling up of evidence based interventions could reduce infection related neonatal deaths by $84 \%$ globally. ${ }^{17}$ This is likely to have a major impact in South Asian countries ${ }^{3}$ because the existing coverage of these interventions in almost all countries is low, except in Maldives and Sri Lanka. For example, the proportion of pregnant women receiving at least four antenatal visits in Afghanistan (18\%), Bangladesh (31\%), India (51\%), Nepal (69\%), and Pakistan (37\%) is low. The proportion of births attended by skilled health workers is $51 \%, 42 \%, 81 \%, 58 \%$, and $55 \%$, respectively. ${ }^{18}$ The median coverage of clean birth practices is $33.9 \%,{ }^{3}$ and of the first postnatal check-up in the first two days of birth is $31 \%$ in all countries except Maldives and Sri Lanka. ${ }^{18}$ Figure 2 lists key interventions that could reduce the high burden of sepsis, sepsis related mortality, and antimicrobial resistance.

Concerted efforts at national and regional levels to identify health system related issues resulting in high incidence of sepsis and antimicrobial resistance, to improve the coverage of known interventions, and to implement context specific solutions that are simple and effective, along with a strong political will, will go a long way to reduce the burden of sepsis related mortality and morbidity in the region.

Contributors and sources: The authors are all engaged in collaborative research for prevention and management of sepsis in neonates. SC, MJS, and RA conceived the idea of the paper with inputs from MS, SS, and SE. SS, RA, and MJS did the literature search while SS extracted the data for systematic review with help from SC. SS and MJS did the analysis. SC and SS wrote the first draft; SS and MJS finalised the manuscript. All the remaining authors contributed to writing/editing various sections, critically reviewing the results, finalising the draft, and approved the final manuscript. MJS is the guarantor.

Competing interests: We have read and understood BMJ policy on conflicts and interests and declare that MS has received research grants from GlaxoSmithKline, Pfizer, and Cubist pharmaceuticals.

Provenance and peer review: Commissioned; externally peer reviewed.

This article is one of a series commissioned by The $B M J$ in collaboration with the Drugs for Neglected Diseases initiative (DNDi). The BM/ retained full editorial control over external peer review, editing, and publication. Open access fees are funded by the DNDi, Geneva.

Suman Chaurasia, consultant ${ }^{1}$

Sindhu Sivanandan, former senior resident ${ }^{1}$

Ramesh Agarwal, professor $^{1}$

Sally Ellis, neonatal sepsis project leader ${ }^{2}$

Mike Sharland, professor $^{3}$

M Jeeva Sankar, assistant professor ${ }^{1}$

${ }^{1}$ Department of Paediatrics, All India Institute of Medical Sciences, New Delhi, India

${ }^{2}$ Global Antibiotic R\&D Partnership, Drugs for Neglected Diseases initiative, Geneva, Switzerland ${ }^{3}$ Paediatric Infectious Diseases Research Group, St George's University London, UK

Correspondence to: M J Sankar jeevasankar@gmail.com

\section{(C) (1) $\Theta$ OPEN ACCESS}

This is an Open Access article distributed in accordance with the Creative Commons Attribution Non Commercial (CC BY-NC 4.0) license, which permits others to distribute, remix, adapt, build upon this work non-commercially, and license their derivative works on different terms, provided the original work is properly cited and the use is non-commercial. See: http://creativecommons.org/licenses/by-nc/4.0/.

$$
\text { Check for updates }
$$

1 GBD 2016 Causes of Death Collaborators. Global, regional, and national age-sex specific mortality for 264 causes of death, 1980-2016: a systematic analysis for the Global Burden of Disease Study 2016. Lancet 2017;390:1151-210. doi:10.1016/ S0140-6736(17)32152-9.

2 Liu L, Oza S, Hogan D, et al. Global, regional, and national causes of child mortality in 2000-13, with projections to inform post-2015 priorities: an updated systematic analysis. Lancet 2015;385:43040. doi:10.1016/S0140-6736(14)61698-6.

3 Das JK, Rizvi A, Bhatti Z, et al. State of neonatal health care in eight countries of the SAARC region, South Asia: how can we make a difference?Paediatrics and International Child Health 2015;35:174-86. doi:10.1 179/2046905515Y.0000000046.

4 Hamer DH, Darmstadt GL, Carlin JB, et al, Young Infants Clinical Signs Study Group. Etiology of bacteremia in young infants in six countries. Pediatr Infect Dis / 2015;34:e1-8. doi:10.1097/ INF.0000000000000549.

5 Saha SK, Schrag SJ, El Arifeen S, et al. Causes and incidence of community-acquired serious infections among young children in south Asia (ANISA): an observational cohort study. Lancet 2018;392:145 59. doi:10.1016/S0140-6736(18)31127-9.

6 World Health Organization. Model list of essential medicines in children. 6th ed. WHO, 2018. 
http://www.who.int/medicines/publications/ essentialmedicines/6th_EMLc2017.pdf.

7 Investigators of the Delhi Neonatal Infection Study (DeNIS) collaboration. Characterisation and antimicrobial resistance of sepsis pathogens in neonates born in tertiary care centres in Delhi, India: a cohort study. Lancet Global Health 2016;4:e75260. doi:10.1016/S2214-109X(16)30148-6.

8 Vergnano S, Menson E, Kennea N, et al. Neonatal infections in England: the NeonIN surveillance network. Arch Dis Child Fetal Neonatal Ed 2011;96:F9-14. doi:10.1136/ adc.2009.178798.

9 Stoll BJ, Hansen NI, Sánchez PJ, et al, Eunice Kennedy Shriver National Institute of Child Health and Human Development Neonatal Research Network. Early onset neonatal sepsis: the burden of group B Streptococcal and E. coli disease continues. Pediatrics 2011;127:817-26. doi:10.1542/ peds.2010-2217.

10 Zaidi AK, Huskins WC, Thaver D, Bhutta ZA, Abbas Z, Goldmann DA. Hospital-acquired neonatal infections in developing countries.
Lancet 2005;365:1175-88. doi:10.1016/S01406736(05)71881-X.

11 Klingenberg C, Kornelisse RF, Buonocore G, Maier RF, Stocker M. Culture-Negative Early-Onset Neonatal Sepsis - At the Crossroad Between Efficient Sepsis Care and Antimicrobial Stewardship. Frontiers in Pediatrics 2018;6:285. doi:10.3389/ fped.2018.00285.

12 Ramasethu J, Kawakita T. Antibiotic stewardship in perinatal and neonatal care. Seminars in Fetal and Neonatal Medicine 2017;22:278-83. doi:10.1016/j. siny.2017.07.001.

13 Patel SJ, Saiman L. Principles and strategies of antimicrobial stewardship in the neonatal intensive care unit. Semin Perinatol 2012;36:431-6. doi:10.1053/j.semperi.2012.06.005

14 Cantey JB, Patel SJ. Antimicrobial stewardship in the NICU. Infect Dis Clin North Am 2014;28:247-61. doi:10.1016/j.idc.2014.01.005.

15 Laxminarayan R, Chaudhury RR. Antibiotic resistance in India: drivers and opportunities for action. PLOS Medicine 2016;13:e1001974. doi:10.1371/journal. pmed.1001974.
16 Llor C, Bjerrum L. Antimicrobial resistance: risk associated with antibiotic overuse and initiatives to reduce the problem. Therapeutic Advances in Drug Safety 2014:5:229-41. doi:10.1177/2042098614554919.

17 Bhutta ZA, Das JK, Bahl R, et al, Lancet Newborn Interventions Review Group, Lancet Every Newborn Study Group. Can available interventions end preventable deaths in mothers, newborn babies, and stillbirths, and at what cost?Lancet 2014:384:347-70. doi:10.1016/ S0140-6736(14)60792-3.

18 United Nations Children's Fund. Maternal and newborn health coverage. Unicef, 2018. https:// data.unicef.org/topic/maternal-health/newborncare/\#status

\section{Appendix: Details of methods and included studies}

Cite this as: BMJ 2019;364:k5314

http://dx.doi.org/10.1136/bmj.k5314 